\title{
Effect of Temporal Modulation on the Local Kinematic Process of Two-Dimensional Chaotic Flow: A Numerical Analysis
}

\author{
M. Telha ${ }^{1,2 \dagger}$, M. Bachiri ${ }^{1}$, Y. Lasbet ${ }^{2}$ and T. T. Naas ${ }^{2}$ \\ ${ }^{1}$ Department of Matter Sciences, Laghouat University, Laghouat, 3000, Algeria \\ ${ }^{2}$ LDMM Laboratory, Ziane Achour University of Djelfa, 17000 Djelfa, Algeria \\ $\dagger$ Corresponding Author Email:telhamostefa@gmail.com
}

(Received January 27, 2020; accepted July 1, 2020)

\begin{abstract}
In this work, a numerical analysis based on CFD method is carried out to examine an unsteady laminar flow of Newtonian fluids in a two-dimensional simulation of a mixer, which is composed of two rods inside a moving cylindrical tank. Three stirring protocols are considered: Non-modulated "NM", Continuously modulated "CM" and non-continuously modulated "ALT" by using the dynamic mesh technique and user defined functions "UDF's" for the velocity profiles. The chaotic advection is obtained by temporal modulation of the rotational velocity of the cylinder and the rods to enhance the mixing of the fluid for very low Reynolds number. For this purpose, we applied the Poincaré map as a reliable mathematic tool to check mixing quality by tracking particles inside the fluid domain. Additionally, we investigated the evolution of local flow proprieties such as rotation rate, deformation rate and elongation rate at different time periods in order to see the effect of temporal modulation on the fluid kinematics. Among the considered protocols, the results of the mentioned simulation showed that it is possible to obtain a chaotic advection only for noncontinuously modulated protocol which enhance mixing fluid efficiency.
\end{abstract}

Keywords: Active mixer; Poincaré map; Deformation; Rotation; Elongation; Chaotic advection; Unsteady flow.

\section{NOMENCLATURE}

$\mathrm{C}_{\mathrm{p}} \quad$ thermal capacity at constant pressure

d tank diameter

D deformation rate

$\mathrm{D}_{\mathrm{m}} \quad$ mean deformation rate

dt time step

$p \quad$ fluid pressure

Pe Peclet number

Pr Prantl number

Re Reynolds number for Newtonian fluid

$\mathrm{R}_{1} \quad$ rod 1 radius

$\mathrm{R}_{2} \quad \operatorname{rod} 2$ radius

$\mathrm{R}_{3} \quad$ tank radius

S total surface of fluid

$\mathrm{t}$ time

$\mathrm{u} \quad$ velocity in the $\mathrm{x}$ direction

$\mathrm{v}$ velocity in the $\mathrm{y}$ direction
$V \quad$ fluid velocity

$\vec{V} \quad$ velocity vector

$\mathrm{x}, \mathrm{y}$ coordinates in Cartesian system

$\varepsilon \quad$ elongation rate

$\rho \quad$ fluid density

$\tau \quad$ time period

$\lambda$ thermal conductivity

$\nabla$ nabla

$\mu \quad$ dynamic viscosity of fluid

$\Omega \quad$ rotation rate

$\Omega_{\mathrm{m}}$ mean rotation rate

$\Omega_{1} \quad$ angular velocity of rod 1

$\Omega_{2} \quad$ angular velocity of rod 2

$\Omega_{3} \quad$ angular velocity of the tank

\section{INTRODUCTION}

Improvement of mixing quality by chaotic advection of Newtonian fluids in confined spaces is used in many engineering fields such as: oil engineering, pharmaceutical plants, petroleum units, and polymer production in chemistry.

The mixing of one or for several fluids may occur 
via hydrodynamic or thermal methods. In each situation, a good comprehension of the physical mechanisms is therefore the key to design an efficient mixing process. The mixing process begins with a "heterogeneous" state and finishes with a "homogeneous" state. The degree of this final homogeneity is related to the efficiency of the mixing process. A mixing state can be considered homogeneous at a big scale, and can be observed non-homogeneous at a small scale El Omari and Le Guer (2010).

The mixing process includes two phases. The first one is known as "stirring phase", in which the different regions of the fluid are scattered, reoriented, sheared, stretched and folded, which can increase the level of the scalar gradient in the fluid. The second phase is the phase where the thermal or molecular diffusion tends to smooth these gradients. Thus, the purpose of the mixing is often to eliminate the concentration or temperature gradients, heat or mass transfers, either by turbulent flows or by laminar chaotic advection.

The mechanism of turbulence is founded on the presence of vortices in the flow which are appearing or disappearing permanently. The big fluctuations in the velocity field leads to the stretching and folding of the fluid particles (Lasbet et al. 2016).

Eckert and Soehngen (1948) observed that, it is possible to acquire an enhanced mixing of a given initial fluid by chaotic advection. He studied the effect of diffusion, conduction and viscosity for reducing the mean value of the given gradient.

(Aref 1984) described a mechanism for the generation of complex Lagrangian trajectories within a fluid, from a relatively simple Eulerian flow. The considered flows are laminar, even noninertial (Stokes flows). Within these flows, initially two adjacent particles have their positions deviate and the distance between them increases exponentially. This property can characterize an efficient mixture and can therefore be exploited in order to mix highly viscous fluids by chaotic advection.

When the flow is generated by moving walls, called active mode (El Omari and Le Guer (2010; Mota et al. 2007; Saatdjian et al. 2011), it provides better control of the flow through the choice of periods, phases, amplitudes and directions of relative wall movements.

In passive mode (when the walls are stationary) (Lasbet et al. 2006,2007; Castelain et al. 2001,2016; Kumar and Nigam(2005)).the chaotic advection is obtained by a suitable arrangement in the space of physical limits of the flow domain.

(Lasbet et al. 2006,2007)used the chaotic advection to enhance both hydrodynamic and thermal performances in different complex geometries for Newtonian fluids. They found that a C-shape configuration represents a higher mixing rate compared to the straight channel.

Kumar and Nigam (2005) characterized fields of flow and temperature in a arrangements of bent coils. The main mechanism generating the flow is the production of spatially chaotic path by varying the path of flow. The bent coil design showed a 20 $30 \%$ improvement in the heat transfer with a low relative pressure drop.(less than 6\%).(Mokrani et al. 1997)analyzed the effect of chaotic advection on thermal process at low Reynolds numbers. In order to evaluate the improvement of heat transfer by chaotic advection, both a helical shell and tube and a chaotic heat exchangers were used. It was indicated that, the heating homogeneity in chaotic flow is practically independent of Reynolds number. Total heat transfer measurements showed that the efficiency of chaotic heat exchanger is greater than the helical one.

Habchi et al. (2009) focused on water/oil dispersions generated by water injection into an oil chaotic advection flow, for low Dean numbers. They showed that chaotic advection causes fluid particles to visit randomly some regions of big elongation and shear rates.

El Omari and Le Guer (2010), studied numerically the improvement of thermal mixing in an active mode (mixer with two rotating rods inside a cylindrical tank) for non-Newtonian highly viscous fluids. Disordered streams were acquired by imposing the temporal modulations.

(Lasbet et al. 2016) studied the effect of the Geometry on the Behavior of the Local kinematic parameters of the Velocity Field in the Laminar chaotic Flow. The results illustrated that a smaller hydraulic diameter is the most appropriate for increasing the local kinematic parameters such as rotation, deformation, stretching and folding.

For non-Newtonian fluids, a numerical study was investigated for a steady laminar flow in two different geometries: complex, called C-shape, and straight channel by (Naas et al. 2016). The generated chaotic flows canupgrade significantlyboth the flow and the thermal performances.

Curved Micromixers were proposed by (BaheriIslami and Khezerloo 2017), to study the behavior of power law fluid under the effect of grooved geometry, for Reynolds numbers ranging from 0.1 to 300 . Their results confirmed that the grooves with $30^{\circ}$ angle enhance well the mixing performance by chaotic advection, although there is no remarkable effect on pressure losses.

(Keshavarzian et al. 2018) studied the improvement of mixing process by means of T-Shape micromixer utilizing a microelectrode structure as a key of chaotic mixing. A set of vortices were created in the working fluid. These vortices especially improve the mixing inside the enclosure.

Another work based on kinematic analysis for Cshape geometry was done numerically by (Naas et al. 2019) using two different fluid temperatures to characterize the thermal mixing rates. Their work proved that the chaotic geometry gave a good performance in terms of thermal homogenization with comparing by the straight channel. 


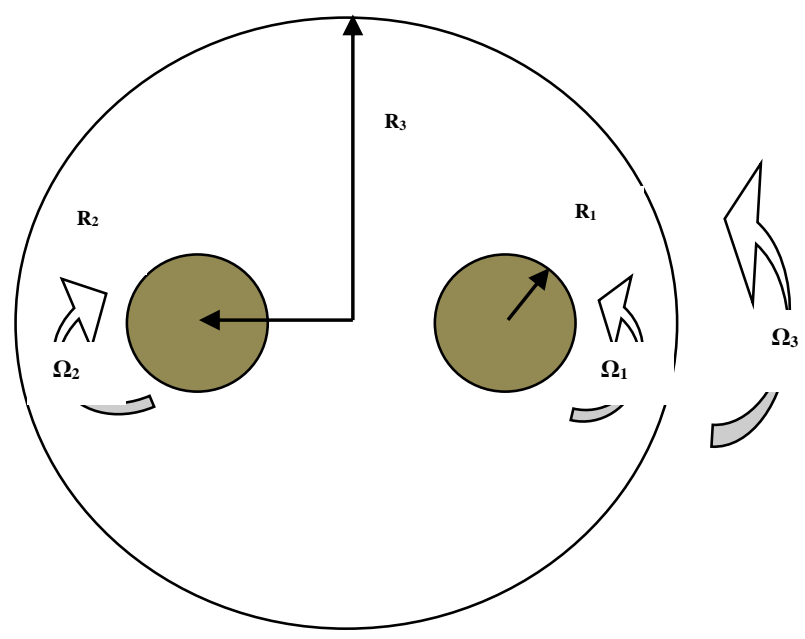

Fig. 1. Geometry of the studied mixer.

Our contribution is to make a comparative dynamic and kinematic analysis, in terms of deformation, rotation and elongation rates of the behavior of Newtonian viscous fluid flowing in an active mixing device as it was used by El Omari and Le Guer (2010) for different stirring protocols (NM,CM and ALT) under a very low Reynolds number.

\section{GEOMETRY, PARAMETERS AND MODELING}

The chosen geometry to carry out these studies on the dynamic chaotic mixture is an active mixer with a simple geometry. It consists of two rods of circular section having the same diameter put in a cylindrical tank as shown in (Fig. 1).

The rods and the tank can rotate around their axes of revolution. The rotations can be steady (NM), continuous (CM) or alternating (ALT). The studied flow is two-dimensional, so the geometrical parameters values are summarized in the table 1 .

\section{Table 1 Geometrical parameters for all cases}

\begin{tabular}{|l|c|c|}
\hline $\mathbf{R}_{\mathbf{1}}$ & Radius of rod 1 & $10 \mathrm{~mm}$ \\
\hline $\mathbf{R}_{\mathbf{2}}$ & Radius of rod 2 & $10 \mathrm{~mm}$ \\
\hline $\mathbf{R}_{\mathbf{3}}$ & Radius of cylindrical tank & $50 \mathrm{~mm}$ \\
\hline
\end{tabular}

Flow conditions and dynamical parameters for every rotation protocol are shown in table 2 shown below.

Mixing by chaotic advection is feasible for low values of Reynolds number.

For our mixing studies, we chose a fluid similar to very viscous oil. The flow in the mixer would be characterized by: a very low Reynolds number, unsteady dynamic boundary conditions and a high Prandtl number.
These dimensionless numbers were chosen as it is shown in the table 3 :

The mass conservation and Navier-Stokes equations were numerically solved using Fluent ANSYS and are given by the following equations respectively:

$\vec{\nabla} \cdot \vec{V}=0$

$\rho \frac{D V}{D t}=-\nabla P+\mu \Delta V$

In the present study, the fluid is considered as Newtonian and incompressible and the fluid flow regime is laminar and unsteady.

The applied boundary conditions for the three stirring protocols are already shown above in table 2.

The SIMPLE scheme is utilized to achieve the pressure-velocity coupling, while in the spatial discretization, a Second-Order upwind scheme is adopted for momentum and second order for pressure.

During the numerical simulations, it is considered that the convergence is achieved when the residues are less than $10^{-4}$ for the conservation equations.

\section{MESHING}

A dynamic grid was used to model the studied fluid flow, because of the domain shape is varying with time due to the motion on the boundaries. For characterization of the motion of two rods \& tank in the model, user-defined functions UDFs as a $\mathrm{C}$ programs were done and inserted in the CFD code.

The size of meshing was chosen after a careful study of independence of the results at the size of the mesh. 
Table 2 Dynamical parameters for all studied protocols

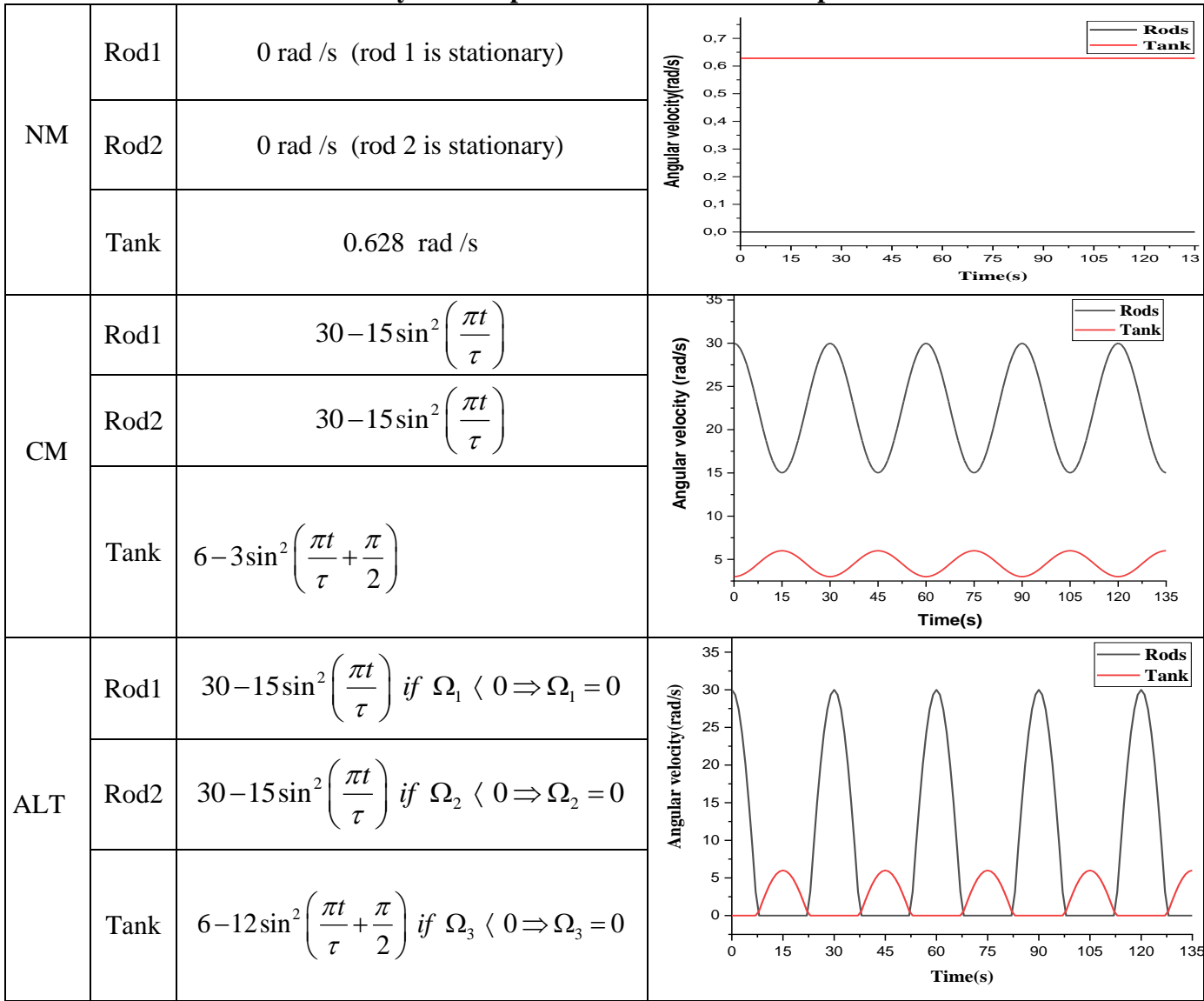

To perform grid independence studies, five grids were used for simulations of Newtonian fluid flow in the active mixer considering an unsteady laminar flow. These grids are ranging from 50 to 90 nodes (step $=10$ ) in the rods edges, and from 200 to 400 in the tank edge ( $\operatorname{step}=50$ ). The velocity magnitude and stream function profiles were evaluated to increase mesh densities.

\section{Table 3 Characteristic numbers for studied flow}

\begin{tabular}{|c|c|c|}
\hline $\begin{array}{c}\text { Reynolds } \\
\text { number }\end{array}$ & $\operatorname{Re}=\frac{\rho V L}{\mu}$ & 1.66 \\
\hline $\begin{array}{c}\text { Peclet } \\
\text { number }\end{array}$ & $P e=\operatorname{Pr} \cdot \operatorname{Re}=\frac{C_{p} \rho V L}{\lambda}$ & $1.610^{4}$ \\
\hline $\begin{array}{c}\text { Prandtl } \\
\text { number }\end{array}$ & $\operatorname{Pr}=\frac{\mu C_{p}}{\lambda}$ & $10^{4}$ \\
\hline
\end{tabular}

Figures 3 and 4 show respectively the evolutions of the velocity magnitude and the stream function versus y-coordinates for various grids at the edges of moving walls. It can be seen that these two quantities profiles are similar and have the same trend for all mesh densities. This illustrates that the velocity magnitude and the stream function are sensitive to the grid mesh except for the mesh densities (80x80x350) and (90x90x400) where no real difference is observed. As conclusion, the (80x80x350) grid which is corresponding to $(10780$ cells) is chosen for computation as an optimal grid mesh.

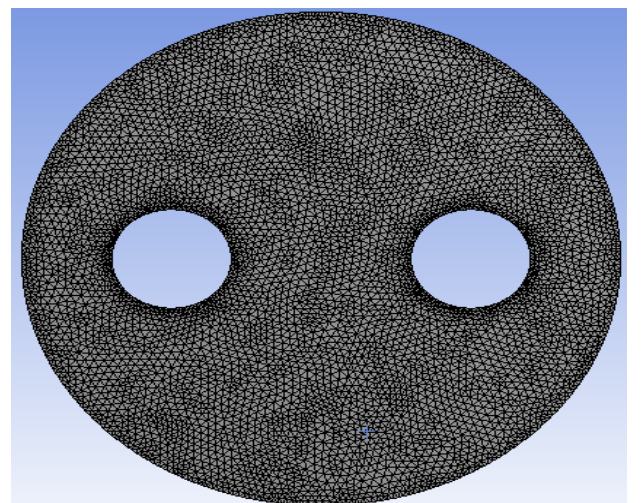

Fig. 2. Mesh of the computation domain inside the mixer ( 10780 cells).

The parameters of the dynamic mesh were chosen carefully as it is schematized below in table 4 , in 
order to make the grid moving with the specified angular velocities already summarized in the table 2 showed above.

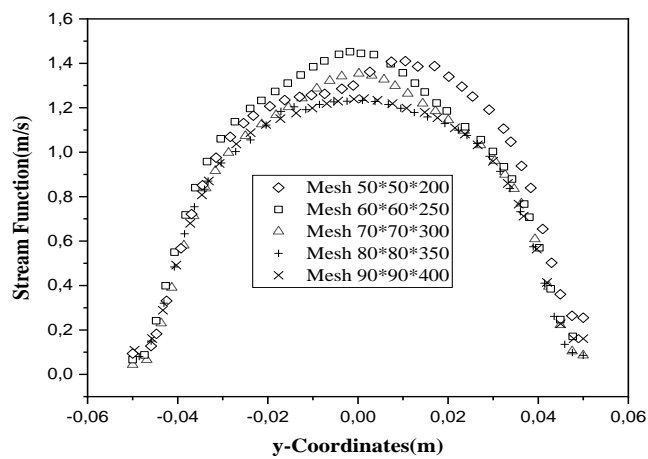

Fig. 3. Velocity magnitude profiles versus $y$ Coordinates for various mesh densities at the edges of moving walls of the mixer.

\section{VALIDATION}

In this section, in order to confirm the precision and the reliability of our calculation software (CFD code), a comparison with some results obtained by (Kamal et al. 2010) using the code (Tamaris). A laminar unsteady flow of Newtonian fluid inside an active mixer is utilized. Figures 3-(a), 3-(b), 3-(c), 3 -(d) present, a comparison of the of streamlines for $\mathrm{CM}$ and ALT stirring protocols at $120 \mathrm{~s}(4 \tau)$ and135s $(4,5 \tau)$. it appears from the figures that there is an almost total stirring protocols at $120 \mathrm{~s}$ $(4 \tau)$ and $135 \mathrm{~s}(4,5 \tau)$. it appears from the figures that there is an almost total agreement between the results.

\section{RESULTS AND DISCUSSION}

\subsection{Flow Characteristics}

Figures (6-a) and (6-b) show the evolutions of the $\mathrm{x}$-velocity and $\mathrm{y}$-velocity profiles with $\mathrm{y}$ and $\mathrm{x}$ coordinates respectively, at the center line of the mixer for different studied stirring protocols (NM, $\mathrm{CM}$ and ALT) in $135 \mathrm{~s}$. Looking at the profiles of the velocity shown below, we can note that, there is an inverted symmetry of velocity for all stirring protocols mentioned above. The maximum and minimum values of velocity are located at the external wall of the tank (about $\pm 0.3 \mathrm{~m} / \mathrm{s}$ ) and it takes almost zero values between the two rods for both NM and ALT stirring protocols, but for CM, the y-velocity is important at the center of mixer. It very well may be seen plainly that the velocity for the NM mode is a bit negligible compared with other protocols. Note also that the axial velocity profiles Figures (6-a) for all protocols have the same tendency especially the CM and the ALT which are superimposed because of the dynamic state of the system at this moment (135s). Returning to Table 2, the tank is moving at the maximum speed in the three protocols which orders the direction and the speed of the fluid.

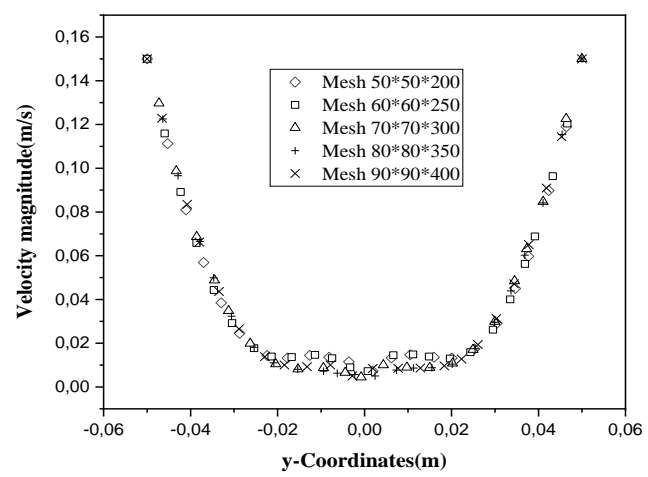

Fig. 4. Stream function profiles versus yCoordinates for various mesh densities at the edges of moving walls of the mixer.

Table 4 Parameters of dynamic grid

\begin{tabular}{|c|c|}
\hline $\begin{array}{c}\text { Method used for } \\
\text { Dynamic grid }\end{array}$ & Settings \\
\hline $\begin{array}{c}\text { Smoothing } \\
\text { Spring/Laplace/B } \\
\text { oundary layer }\end{array}$ & $\begin{array}{c}\text { Spring constant factor : 0.3 } \\
\text { Convergence tolerance }: 0.001 \\
\text { Number of iterations : } 20 \\
\text { Laplace node relaxation : } 1\end{array}$ \\
\hline $\begin{array}{c}\text { Remeshing local } \\
\text { cell }\end{array}$ & $\begin{array}{c}\text { Minimum length scale }: 0.0007 \\
\text { Maximum length scale }: 0.002 \\
\text { Maximum cell skewness : } 0.47 \\
\text { size remeshing interval :1 }\end{array}$ \\
\hline Refinement & 1 \\
\hline Method & triangles \\
\hline Sizing of rods & 80 \\
\hline Sizing of tank & 350 \\
\hline
\end{tabular}

As it is shown For the NM stirring protocol (no modulation applied) in Fig.5.(a). It is characterized by aperiodic set of regular streamlines alongside the wall of the rotating tank, a stagnation point at the focal point of the mixer and two big vortices are created. Velocity vectors therefore have the same topology as it is illustrated in Fig. 5. (b). the arrows show the direction of the rotation tank (counter clockwise) For CM stirring protocol at $\mathrm{t}=135 \mathrm{~s}(4.5 \tau)$ i.e. the external wall is moving at its max velocity. We see that new recirculation regions are generated, cause the rise of two vortices of high velocity around it Fig. 6. (a).It is so clear that velocity vectors show us the fluid direction in the domain as it is shown in Fig. 6. (b).

In the case of non-continuously modulated stirring protocol (ALT) Fig. 7. (a), the streamlines of stream function are similar in topology to those of non-modulated stirring protocol (NM) because of at $135 \mathrm{~s}$ it just the tank which is in rotation under a specified angular velocity above-mentioned. Velocity vectors Fig. 7. (b) show the flow direction and the regions of high velocity inside the mixer. 

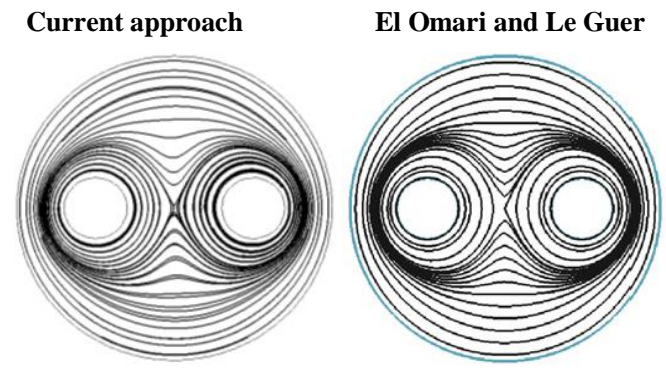

(a)

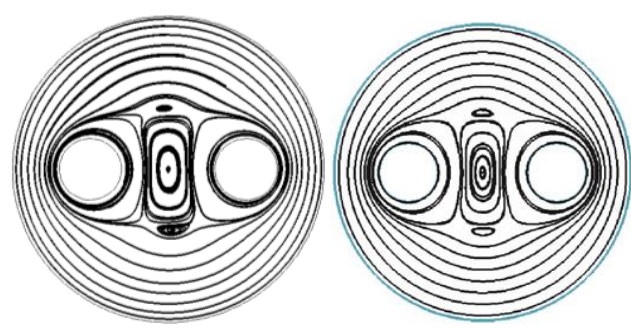

(b)
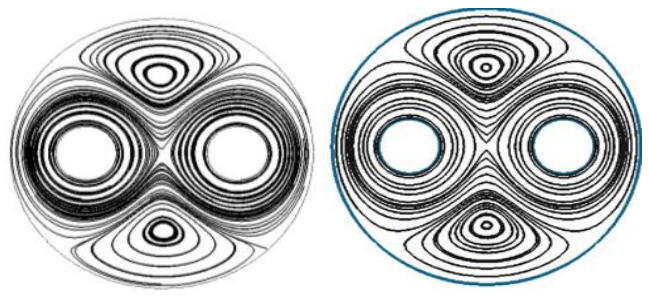

(c)
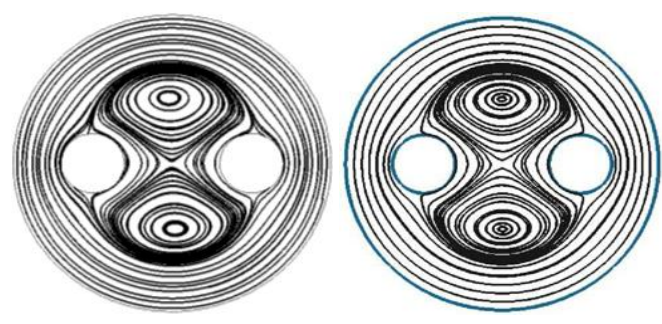

(d)

Fig. 5. Streamlines of stream function for the CM stirring protocol at (a): $4 \tau(120 \mathrm{~s})$ of modulation(b):4.5 $\tau$ (135 s) of modulation and for ALT stirring protocol at (c):4 4 (120 s) of modulation (d): $4.5 \tau$ (135 s) of modulation.

\subsection{Poincaré Section}

A Poincaré section is a mathematical analysis tool which is applied to characterize the chaos and to study the dynamic of a system. It is based on tracking some selected particles starting from their initial positions. If the initial positions are properly chosen (Yuan and Isaac 2017), they will indicate the behavior of whole fluid domain. After the choice of initial points, the new particle positions at the end of each time period will be saved on file and then graphed on one map. In general, computing Poincaré section can give a 'regular' or a 'chaotic' behaviors. A 'regular' behavior means that the particle trajectories are nearly superposed to regular orbits. the 'chaotic' behavior is indicated when the particle positions are scattered all over the fluid domain. It is known that a Poincaré map shows some regions where the initially put particles pass upon or are not allowed from entering regardless of time periods number. If the behavior is chaotic, the forbidden areas become smaller and disappear at the end with increasing time periods numbers. To carry out Poincaré maps (Habchi et al. 2009), five points from fluid domain are selected initially (at $\mathrm{t}=0 \mathrm{~s}$ ) as follows: $\mathrm{P} 1(\mathrm{x}=0, \mathrm{y}=0.01) \mathrm{mm}, \mathrm{P} 2(\mathrm{x}=0, \mathrm{y}=0.02)$ $\mathrm{mm}, \mathrm{P} 3(\mathrm{x}=0, \mathrm{y}=0.03) \mathrm{mm}, \mathrm{P} 4(\mathrm{x}=0, \mathrm{y}=0.04)$ $\mathrm{mm}$ and $\mathrm{P} 5(\mathrm{x}=0, \mathrm{y}=0.05) \mathrm{mm}$ Fig. 8. It shows the Poincaré maps at different time periods $\mathrm{T}=30 \mathrm{~s}$, $60 \mathrm{~s}, 90 \mathrm{~s}, 120 \mathrm{~s}$ and $135 \mathrm{~s}$, for non-modulation stirring protocol (NM), we see for all five chosen points, a clear regular and similar behaviors regardless of time periods, which is interpreted by the constancy of the external moving tank speed (i.e. no modulation) and the zero speed of two internal agitator rods, hence coinciding streamlines and path-lines are obtained. For the second stirring protocol called Continuous modulation (CM), we note that for all time periods, some chaotic areas around and between the two rotating rods (from mixer center till the level of P3), on the other side some forbidden regions are noticed especially around the wall of external tank (from the level of P3 till the level of P5). The third stirring protocol which is named Alternately modulated (ALT), the fluid domain becomes more and more chaotic since the first time period ( $\mathrm{T}=30 \mathrm{~s})$, the particles begin to scatter all over the regions, the regular trajectories commence to become more and more chaotic by increasing time periods. Practically, at ( $T=135 \mathrm{~s})$, it is notable that there are not forbidden regions almost. It is the effect of the alternation of the two rods and the tank that creates the chaos to the flow.

\subsection{Local Properties}

In order to assess the performance of the considered stirring protocols in this investigation, we introduce several kinematic concepts related to flowing fluids.

\subsection{1.a Mean Deformation Rate}

Deformation process is always present in the mixing operations. it achieves an enhanced mixing quality by molecular diffusion. For this goal, the modulation in time is a reliable solution for increasing the deformation rate.

It is defined by the following relation(Khakhar and Ottino 1986) :

$D=\left[2\left(\frac{\partial u}{\partial x}\right)^{2}+2\left(\frac{\partial v}{\partial y}\right)^{2}+\left(\frac{\partial u}{\partial y}+\frac{\partial v}{\partial x}\right)^{2}\right]^{\frac{1}{2}}$

In the present study, Evolutions of the mean value of deformation rates $\left(D_{\mathrm{m}}\right)$ of the three considered stirring protocols (NM, CM and ALT) in a constant Reynolds number $(\operatorname{Re}=1.66)$ calculated at $135 \mathrm{~s}$, are presented in Fig. 8 


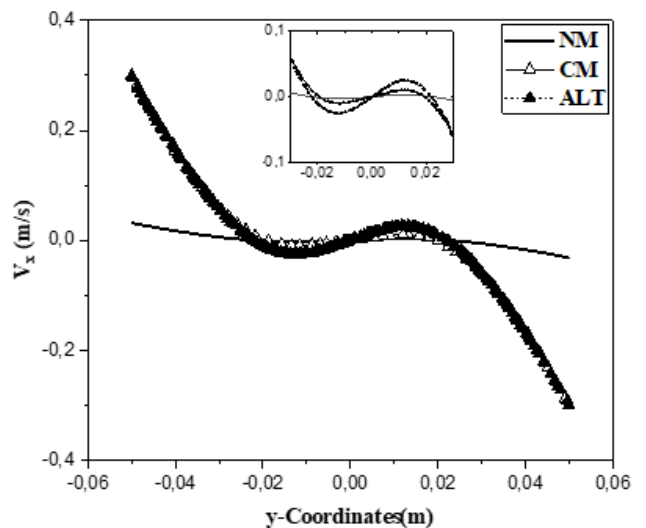

(a)

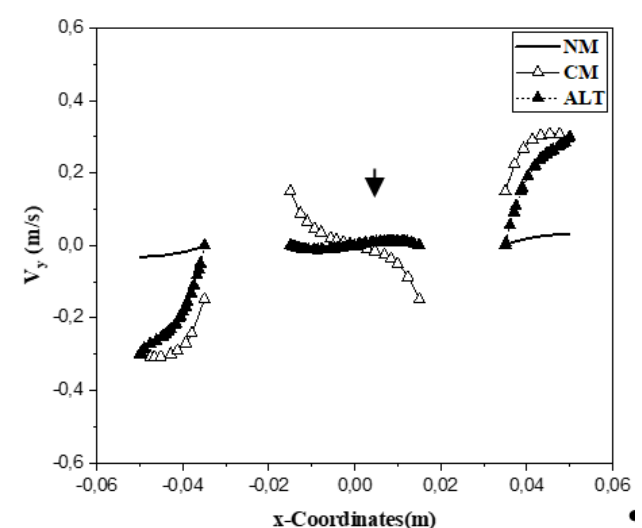

(b)

Fig. 6. Velocity profiles at $135 \mathrm{~s}(4,5 \mathrm{~T})$ for different studied stirring protocols (a) $\mathrm{x}$-component of velocity versus. $y$ (b) $y$-component of velocity versus. $x$.

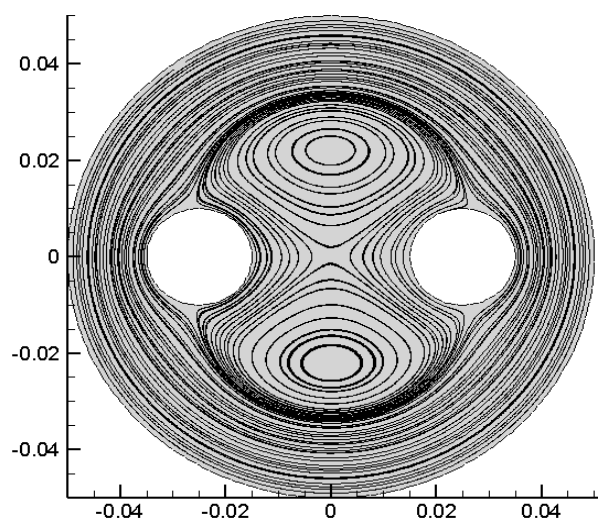

(a)

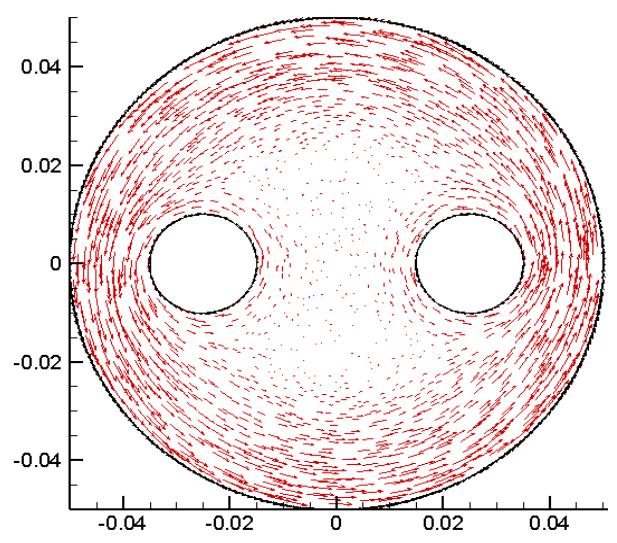

(b)

Fig. 7. (a) Streamlines of stream function (b)Velocity vectors. For NM stirring protocol at $135 \mathrm{~s}$.

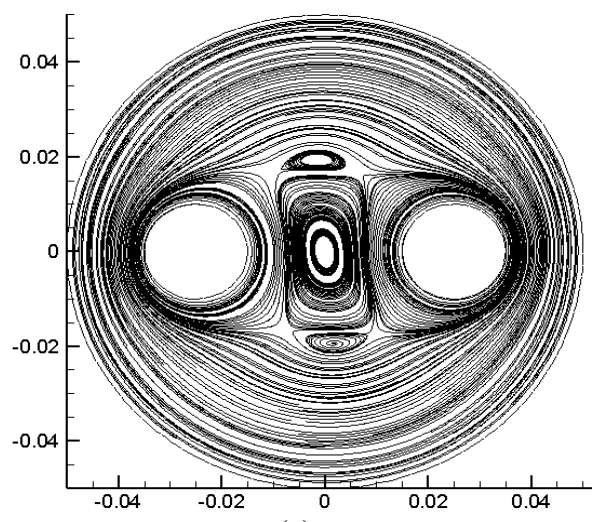

(a)

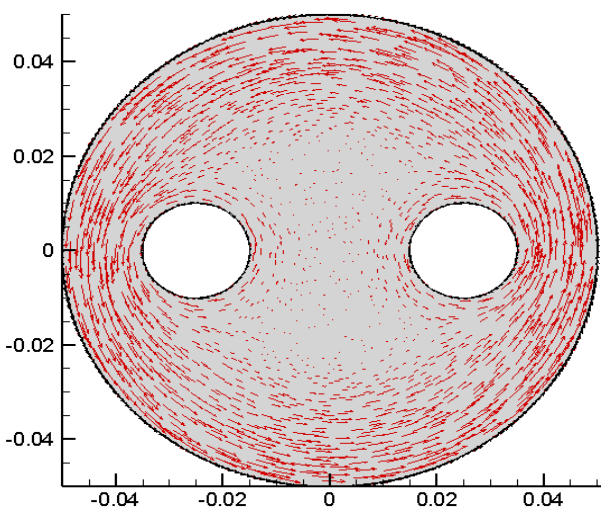

(b)

Fig. 8. (a) Streamlines of stream function (b) Velocity vectors. For CM stirring protocol at $135 \mathrm{~s}$.

$D_{m}=\frac{1}{S} \int D \mathrm{dS}$

This parameter is more important, and the flow becomes more agitated and sheared. the flow in $\mathrm{CM}$ and ALT cases (when temporal modulation is applied) has a high deformation rate compared to the NM one (no temporal modulation is applied) in both x-coordinates and y-coordinates Figs. 8(a) and (b) especially near the external wall of the tank for ALT case and near the walls of rods and 


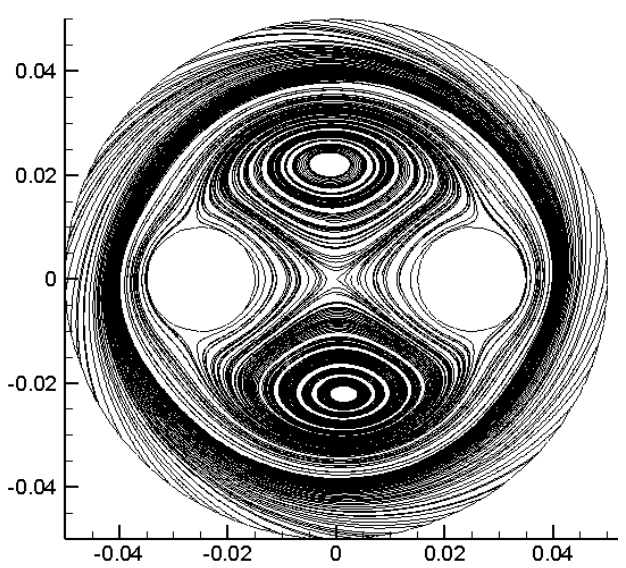

(a)

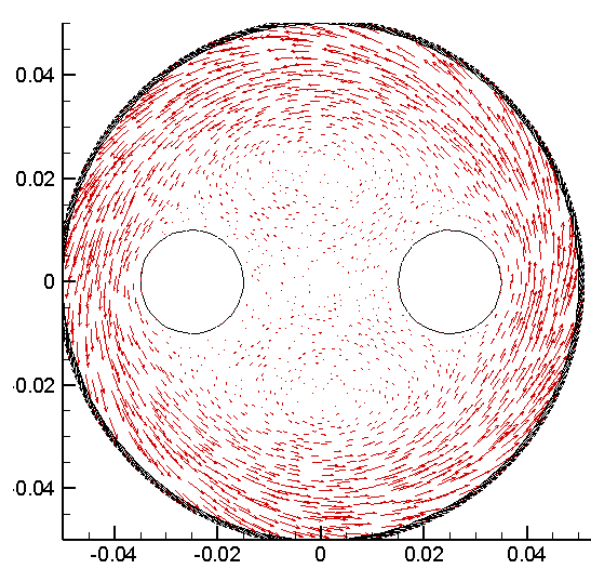

(b)

Fig. 9. (a) Streamlines of stream function (b) Velocity vectors. For ALT stirring protocol at $135 \mathrm{~s}$.

external wall of the tank for $\mathrm{CM}$ case. This explains that the kinematic behavior is accentuated by the effect of temporal modulation.

\subsection{1.b Instantaneous Deformation Rate}

By using CFD fluent, the deformation rates are calculated at every half period $(15 \mathrm{~s}, 30 \mathrm{~s}, 45 \mathrm{~s}, 60 \mathrm{~s}$, $75 \mathrm{~s}, 90 \mathrm{~s}, 105 \mathrm{~s}, 120 \mathrm{~s}, 135 \mathrm{~s}$ ) in order to control the behavior of the flow and to know which relation can be exist between the deformation and the stirring protocol and also the modulation in time Fig. 9.

The results obtained show that this mechanism can increase well influenced by the rotation of both the rods and the tank at every chosen instant. in $\mathrm{CM}$ case $\left(9.4 \mathrm{~s}^{-1}<\mathrm{D}<11.22 \mathrm{~s}^{-1}\right)$, the two rods are rotating together in the pair half periods and the tank is stopped. but in ALT case,the tank is rotating all alone in impair half periods $\left(8.13 \mathrm{~s}^{-1}<\mathrm{D}<10.28 \mathrm{~s}^{-1}\right)$ and the rods are stopped.in NM case the deformation rate take an approximate value of $0.8812 \mathrm{~s}^{-1}$

\subsection{2.a Mean Rotation Rate}

In general, the fluid particle rotates as it deforms and shears, and the rate of rotation is calculated according to the definition given in the following relation:

$\Omega=\frac{1}{2}\left(\frac{\partial v}{\partial x}-\frac{\partial u}{\partial y}\right)$

In our present study, Evolutions of the mean value of rotation rates $\left(\Omega_{\mathrm{m}}\right)$ for the three studied stirring protocols (NM, CM and ALT) in a low Reynolds number $(\mathrm{Re}=1.66)$ calculated at $135 \mathrm{~s}$, are shown in Fig. 10.

$\Omega_{m}=\frac{1}{S} \int \Omega \mathrm{dS}$

we note according to the diagram presented above that the rate of rotation increases in the vicinity of the walls of the external tank and in the area between the cylindrical rods and this for the case of
$\mathrm{CM}$ stirring protocol. in the other side for the case ALT, the rate of rotation is more important but only in the vicinity of walls of the external tank. the rate in question is relatively low for the NM case in the vicinity of walls of tank and tends towards a zero value between the two rods .

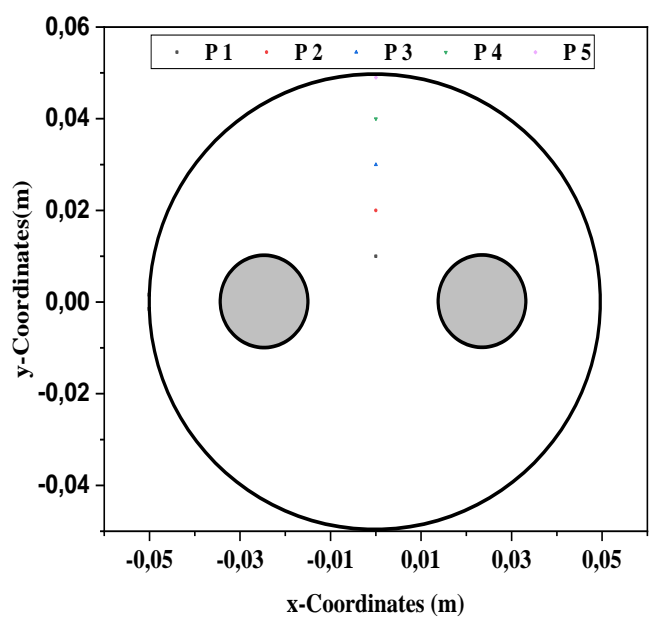

Fig. 10. Initial chosen points for Poincaré section.

\subsection{2.b Instantaneous Rotation Rate}

Counter to the deformation case, the rotation rate is higher in the impair half-periods for the ALT case $\left(\Omega \max =13.6 \mathrm{~s}^{-1}\right)$. and for pair ones, it is the CM which is more important Fig. 11.

And for NM stirring protocol, the values of instantaneous rotation rates are nearly similar and low for all taken times $\left(\Omega=1.45 \mathrm{~s}^{-1}\right)$.

\subsection{3.a Mean Elongation Rate}

The generalized elongation rate $\varepsilon$ given by Eq. (7), is determined analytically from the derivatives of the velocity components, specified in (Germain 1962) 


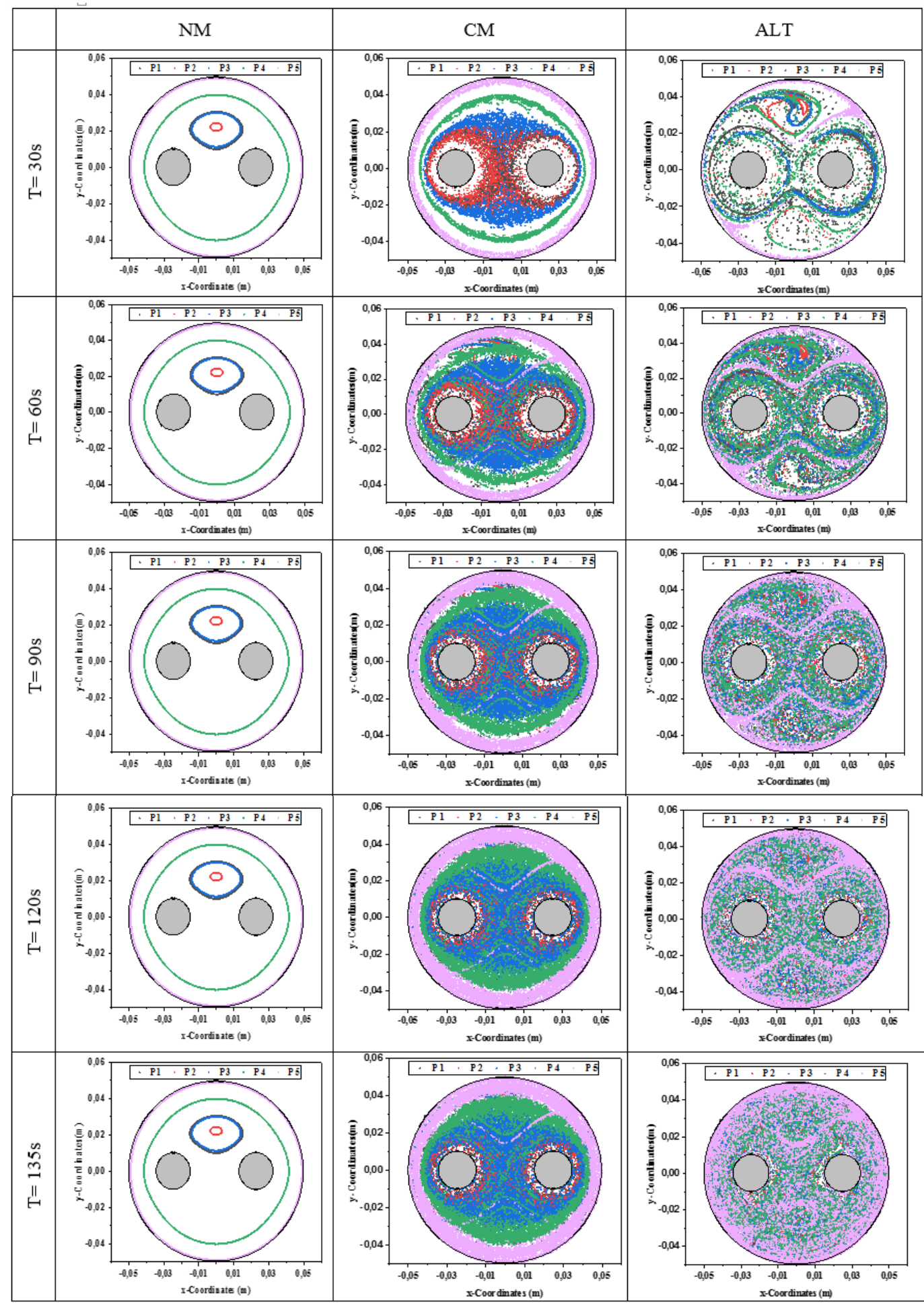

Fig. 11. Evolution of poincaré section for NM, CM and ALT stirring protocols at different periods $(1 \tau, 2 \tau, 3 \tau, 4 \tau, 4.5 \tau) \quad(\tau=30 \mathrm{~s})$.

and in (Bird et al. 2007), and defined, as the second invariant of the extension along theaxis. This expression is also used by (Khakhar and Ottino 1986).

$$
\varepsilon=\frac{u^{2}\left(\frac{\partial u}{\partial x}\right)+v^{2}\left(\frac{\partial v}{\partial y}\right)+u . v\left(\frac{\partial u}{\partial y}+\frac{\partial v}{\partial x}\right)}{u^{2}+v^{2}}
$$




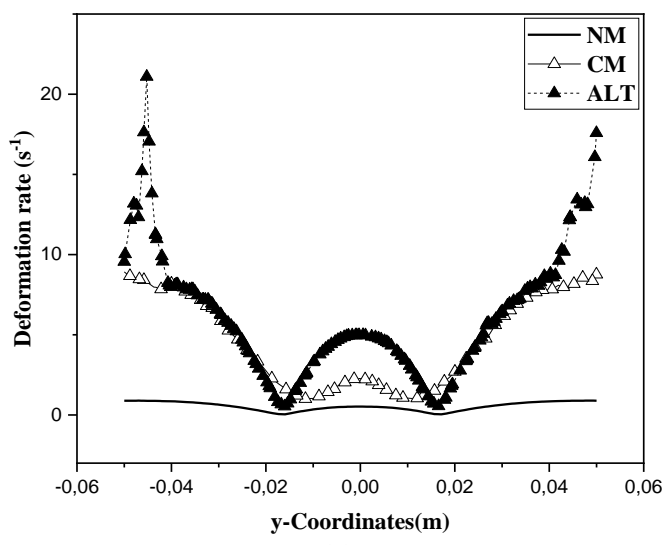

(a)

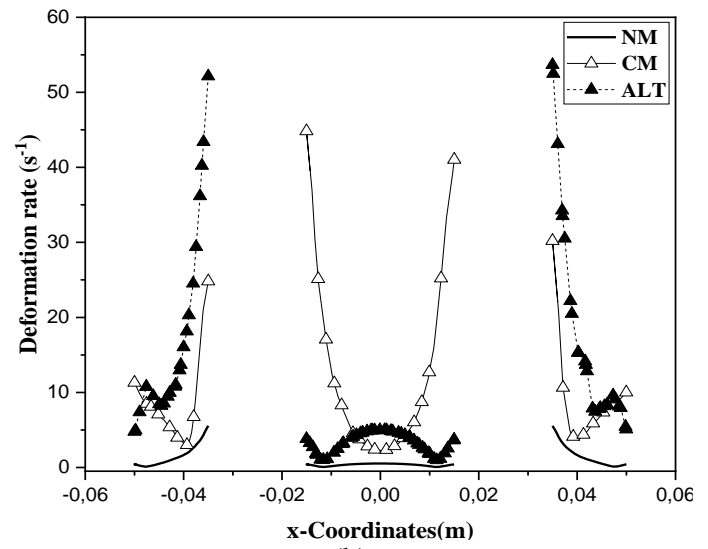

(b)

Fig. 12. Computed deformation rates in the mixer at $135 \mathrm{~s}(4.5 \tau)$.

he CFD code allowed to calculate the elongation rates for all studied stirring protocols. These elongation rates can be positives or negatives (contraction)

According to scheme shown below Fig. 12, we note for $\mathrm{x}$-coordinates that the $\mathrm{CM}$ stirring protocol and the ALT one are nearly symmetric in shape, fluid particles near the external wall are elongated for ALT mode and contracted for CM mode and the contrary for the region that is between two rods. For $\mathrm{NM}$ case, the elongation rate takes a very low value.

For y-coordinates, we note that there are some similarities and superposition of two curves (CM\&ALT) .but always NM stirring protocol gives a zero value of this rate.

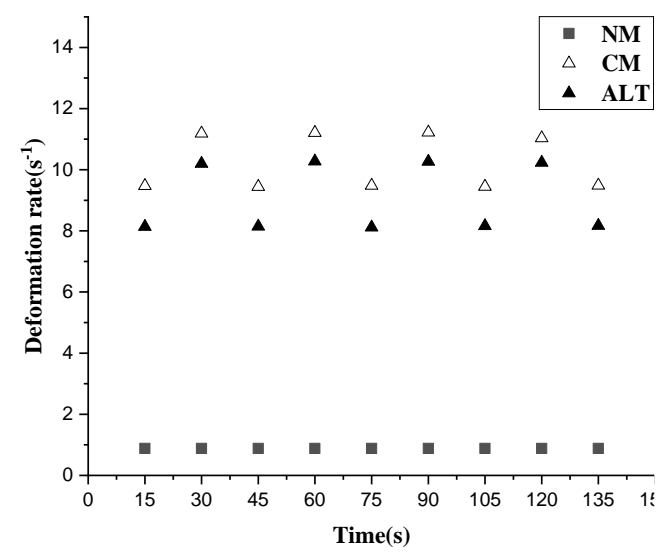

Fig. 13. Evolution in time of deformation rates of whole fluid domain.

\subsection{3.b Instantaneous Absolute Elongation Rate}

For the whole fluid, we see according to Fig. 11 that the most values of this property are around the zero value mainly for the protocols (NM\&CM), but ALT stirring protocol showed considerable values especially at $90 \mathrm{~s}$ when this value reaches $2.46 \mathrm{~s}^{-1}$.

\section{CONCLUSION}

A careful numerical study was carried out by using Fluent ANSYS, in order to investigate the effect of angular velocity modulated in time on the enhancement of mixing and the variation of local kinematic properties of the fluid flow at very low Reynolds number. Three different stirring protocols were studied: NM, CM and ALT. The chaotic advection can be described by Poincaré section as a very reliable mathematical tool. The fluid flow properties which can be characterized are: deformation (strain rate), rotation (vorticity magnitude) and elongation rate. The maps obtained by Poincaré section method exhibited a net chaotic state for ALT stirring protocol after $135 \mathrm{~s}$, this time represents the established time in which after this value, the results are similar. But the other two protocols are regular according to the maps obtained.

Local properties of the studied flow in terms of rotation, deformation rates are important for both $\mathrm{CM}$ and ALT stirring protocols; although CM showed better kinematic parameters values compared to ALT one. These results indicate a relative chaotic regime of the fluid flow. In addition, the ALT stirring protocol revealed a great elongation compared to the others (NM and $\mathrm{CM}$ ). As it is known, the enhancement of these said kinematic properties (deformation, rotation and elongation rates) in the fluid flow can considerably improve the mixing level of the fluids.

As a conclusion, the study explored the effect of the temporal modulation on the improvement of local kinematic process of the fluid flow in terms of rotation, deformation and elongation and it was found that ALT stirring protocol showed higher values.

The obtained results of our study can be used in many processes and for multiple industrial applications, which the aim is to obtain an homogeneous state in a reduced time and 

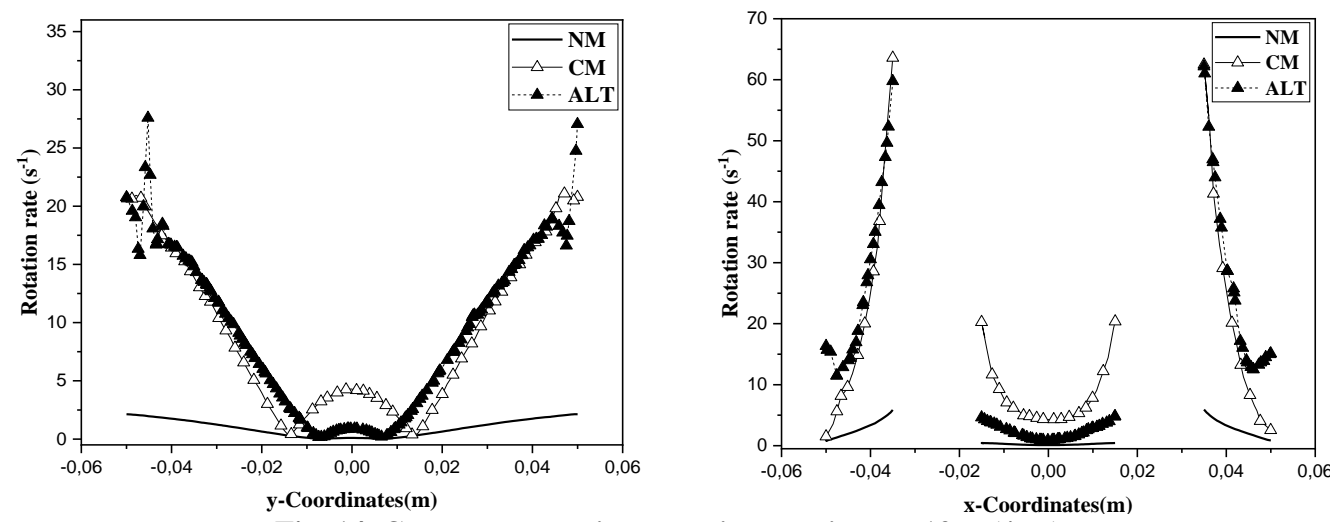

Fig. 14. Computed rotation rates in the mixer at $135 \mathrm{~s}(4.5 \tau)$.

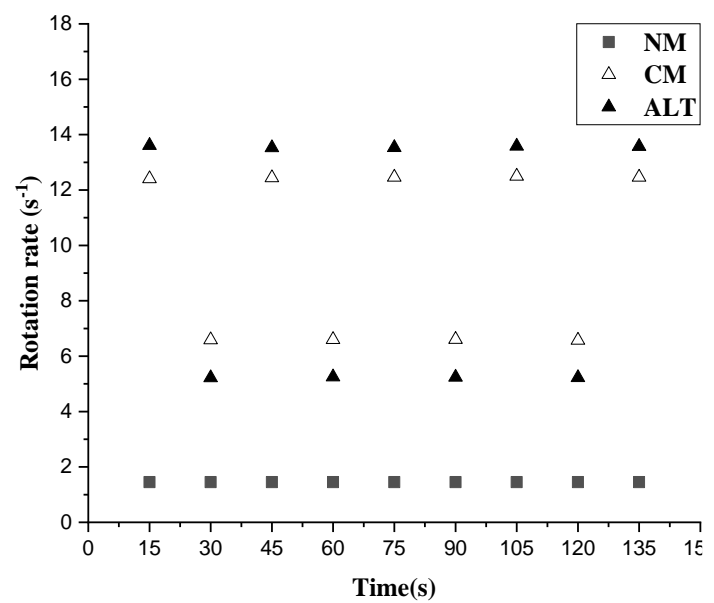

Fig. 15. Evolution in time of rotation rates of whole fluid domain.
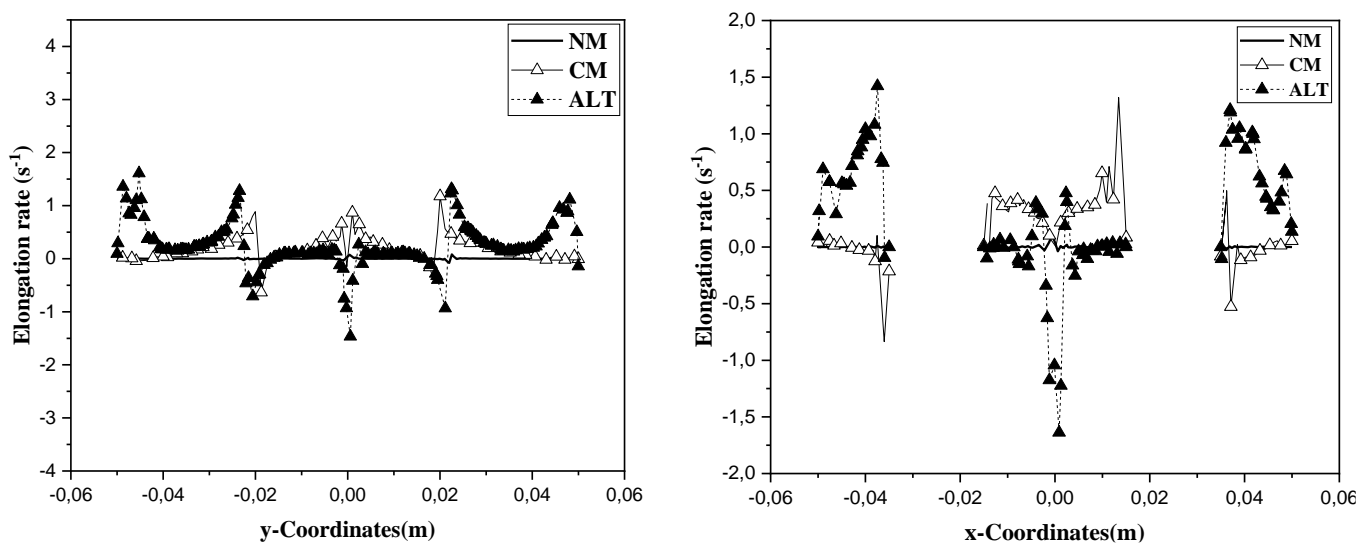

Fig. 16. Computed elongation rates in the mixer at $135 \mathrm{~s}(4.5 \tau)$. 
consequently with low power consumption. For instance: the production of emulsions, the manufacture of food products and pharmaceutical products, as it can be used for the production and mixing of paint.

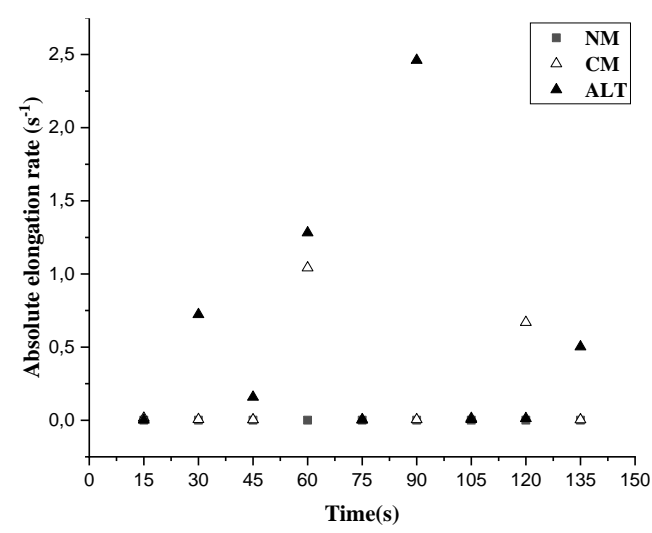

Fig. 17. Evolution in time of absolute elongation rates of whole fluid domain.

\section{REFERENCES}

Aref, H. (1984). Stirring by chaotic advection. $J$ FluidMech 143, 1-21.

BaheriIslami, S. and M. Khezerloo (2017). Enhancement of Mixing Performance of NonNewtonian Fluids using Curving and Grooving of Microchannels. Journal of Applied Fluid Mechanics 10 (1), 127-141.

Bird, R. B., W. E. Stewart and E. N. Lightfoot (2007). Transport Phenomena. Wiley, New York.

Castelain, C., A. Mokrani, Y. Le Guer and H. Peerhossaini (2001). Experimentaly study of chaotic advection regime in a twisted duct flow. European Journal of Mechanics B Fluids 20, 205-232.

Castelain, C., Y. Lasbet, B. Auvity and H. Peerhossaini (2016). Experimental study of the thermal performance of chaotic geometries for their use in PEM fuel cells. International Journal of Thermal Sciences 101, 114-191.

Eckert, E. R. G. and E. Soehngen (1948). Studies on Heat Transfer in Laminar Free Convection with Zehnder-Mach Interferometer. USAF Technical Report, 5747.

El Omari, K. and Y. Le Guer (2010). Alternate rotating walls for thermal chaotic mixing.International Journal of Heat and Mass Transfer 53, 1-3, 123-134.

El Omari, K., T. Kousksou and Y. Le Guer (2011). Impact of shape of container on natural convection and melting inside enclosures used for passive cooling of electronic
devices.Applied Thermal Engineering 31,3022-3035.

Germain, P. (1962). Mécanique des milieux continues. Ed. Masson.

Habchi, C., T. Lemenand, D. Della Valle and H. Peerhossaini (2009). Liquid-liquid dispersion in a chaotic advection flow. In J Multiphase Flow 35, 485-497.

Keshavarzian, B., M. Shamshiri, M. Charmiyan and A. Moaveni (2018). Optimization of an Active Electrokinetic Micromixer Based on the Number and Arrangement of Microelectrodes. Journal of Applied Fluid Mechanics 11(6), 1531-1541.

Khakhar, D. V. and J. M. Ottino (1986). Deformation and breakup of slender drops in linear flows. J FluidMech 166, 265-285.

Kumar, V. and K. D. P. Nigam (2005). Numerical simulation of steady flow fields in coiled flow inverter. International Journal of Heat Mass Transfer 48, 4811-4828.

Lasbet, Y., A. Bruno, C. Cathy and H. Peerhossani (2007). Thermal and Hydrodynamic Performances of Chaotic Mini-Channel: Application to the Fuel Cell Cooling. J. Heat Tran Eng 28(8), 795-803.

Lasbet, Y., B. Auvity, C. Castelain and H. Peerhossani (2006). A chaotic heat-exchanger for PEMFC cooling applications. Journal of Power Sources 156, 114-118.

Lasbet, Y., L. Aidaoui and K. Loubar (2016, September). Effects of the Geometry Scale on the Behaviour of the Local Physical Process of the Velocity Field in the Laminar Flow. International Journal of Heat And Technology 34(3)439-445.

Mokrani, A., C. Castelain and H. Peerhossaini (1997). The effects of chaotic advection on heat transfer. International Journal of Heat and Mass Transfer, 40(13), 3089-3104.

Mota, J. P. B., A. J. S. Rodrigo and E. Saatdjian (2007). Optimization of heat-transfer rate into time periodic two-dimensional Stokes flows. International Journal for Numerical Methods in Fluids 53(6), 915-931.

Naas, T. T., Y. Lasbet, A. Benzaoui and K. Loubar (2016). Characterization of Pressure Drops and Heat Transfer of Non-Newtonian Power-Law Fluid Flow Flowing in Chaotic Geometry. International Journal of Heat and Technology 34(3), 251-260.

Naas, T. T., Y. Lasbet, L. Aidaoui, A. L. Boukhalkhal and K. Loubar (2019). High performance in terms of thermal mixing of non-Newtonian fluids using open chaotic flow: numerical investigations. International Journal of Heat and Technology 34(3), 251-260.

Saatdjian, E., A. J. S. Rodrigo and J. P. B. Mota (2011). Stokes flow heat transfer in an annular, 
M. Telha et al. / JAFM, Vol. 14, No. 1, pp. 187-199, 2021.

rotating heat exchanger. Applied Thermal Engineering 31(8-9),1499-1507.

Tohidi, A., S. M. Hossein Ali Pour, M. Shokrpour, and A. S. Mujumdar (2015). Heat transfer enhancement utilizing chaotic advection in coiled tube heat exchangers. Applied Thermal
Engineering 76, 185-195.

Yuan, F. and K. M. Isaac (2017). A study of MHDbased chaotic advection to enhance mixing in microfluidics using transient three-dimensional CFD simulations. Sensors and Actuators $B$ 238, 226-238. 